\title{
Welcome to Raman Spectroscopy: Successes, Challenges, and Pitfalls
}

Jill Dill Pasteris ${ }^{1}$ and Olivier Beyssac ${ }^{2}$

${ }^{1}$ Washington University in St. Louis, Department of Earth and Planetary Sciences and Institute for Materials Science and Engineering, 1 Brookings Dr., CB 1169, St. Louis, Mo 63130-4899; pasteris@levee.wustl.edu

2 Institut de Minéralogie, de Physique des Matériaux et de Cosmochimie (IMPMC), UMR 7590 Sorbonne Université, CNRS \& MNHN, Campus Jussieu, 4 place Jussieu, 75005 Paris, France (Olivier.Beyssac@upmc.fr)

\begin{abstract}
Geoscientists quickly recognized the broad applicability of Raman microprobe spectroscopy to the Earth and planetary sciences, especially after commercially built microprobe instruments became available in the early 1980's. The sensitivity of Raman spectra to even minor (chemical or structural) perturbations within chemical bonds in (even amorphous) solids, liquids, and gases has been applied to the identification, characterization, and differentiation of, e.g., individual minerals, fluid inclusions, glasses, carbonaceous materials, solid solution phases, strain in minerals, and dissolved species in multi-component solutions. The articles in this issue explore how Raman spectroscopy has deepened and broadened our understanding of geological and extraterrestrial materials and processes.
\end{abstract}

KEYWORDS: Raman spectroscopy, Earth sciences, applications, spectrometers, spectra, minerals 


\section{INTRODUCTION}

Raman spectroscopy is an analytical technique strongly suited to application in the Earth and planetary sciences, chemistry, materials science, medicine, and industry. Ongoing improvements in several components of Raman systems (e.g., lasers, computers, detectors, motorized stages, software) have encouraged much innovation in instrumentation (Adar 2001; Dubessy et al. 2012), both broadening the range of instrumental capabilities (e.g., improved spectral imaging/mapping, field-portable Raman instruments, hybrids such as Raman-atomic force microscope) and targeting of specialized needs (e.g., undersea applications, deployment on Mars) (Dubessyet al. 2012; Cardell and Guerra 2016; Chou and Wang 2017; Beyssac this issue). The Raman effect was discovered/demonstrated in 1928 by Chandrasekhara Venkata Raman and Kariamanickham Srinivasa Krishnan (1930 Nobel prize in physics to Raman for this discovery) and has been routinely used in physics and chemistry since the 19501960s following mainly the development of laser sources and photon detectors. In Earth sciences, Raman spectroscopy became popular later in the 1980s with the development and commercial availability of Raman microprobes. Since then, as happened with isotope analysis and electron probe microanalysis decades earlier, earth scientists have been quick to pursue new applications of Raman spectroscopy, which in turn has helped to reinvigorate or to open up multiple fields of research, such as fluid-inclusion-based studies, the search for traces of life in the oldest terrestrial rocks or extraterrestrial materials, and mineralogy of the deep Earth by in situ characterization in diamond-anvil cells. Another major early and continuing use has been in gemology for the identification of gems and the mineral and fluid inclusions in them (e.g., for verification of natural origin).

This article and more generally this issue provide no learner's handbook of Raman spectroscopy, firstly for lack of space, but also because there are many books and articles that address the physics of the Raman effect and how it can be exploited in the context of specific instrumentation and analytical techniques. Instead, we offer here a basic-level overview of "how Raman works" in order to alert readers to which types of samples and questions Raman spectroscopy typically can be applied. Some 
of the information below will be understood better in the context of individual articles in this issue.

\section{RAMAN EFFECT IN A FEW PARAGRAPHS}

Raman spectroscopy deals with a scattering phenomenon in which photons (from a laser) of a single quantum energy are focused/collimated by a lens onto or into a sample. After interaction with the atoms of the sample, most importantly their covalently bonded components, some portion of the resultant back-scattered photons is captured by the same lens, transmitted and dispersed through a spectrometer, then digitized according to wavelength via a detector (Fig. 1). Those photons returning with the same quantum energy as that of the laser (i.e., Rayleigh elastic scattering) are not of interest here, but rather those (only 1 in $10^{7}$ ) that return with quantum energies shifted from that of the laser source (Fig. 1). The amounts of those shifts, recorded as wavenumber values (i.e., $\Delta \mathrm{cm}^{-1}$, the "Raman shift" due to inelastic scattering), reflect the energies of the vibrations between bonded atoms within the internal structure/symmetry of the host material.

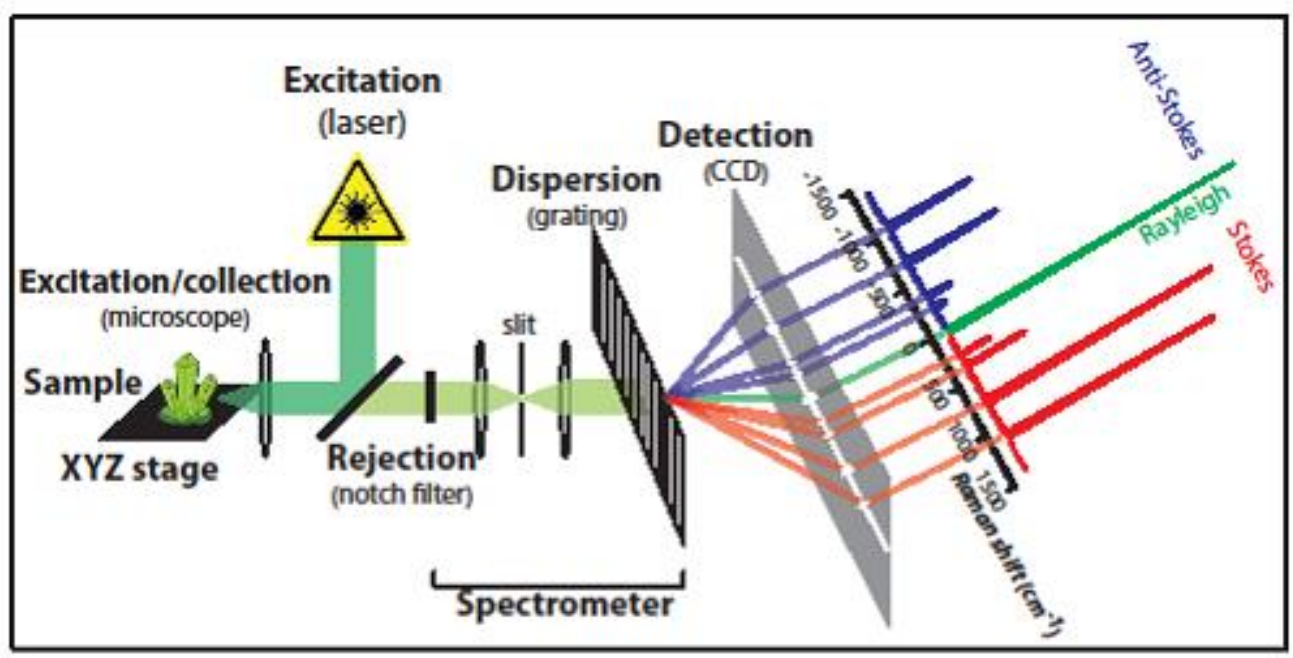

Figure 1: Schematic overview of the basic instrumental setup required to take a Raman spectrum. Note that one typically views the Stokes-scattering (red-shifted, i.e.,right-hand) portion of the spectrum. 
Photons in the ultraviolet (UV) to infrared (IR) range of the electromagnetic spectrum may transfer energy to molecules or crystals as vibrations or electronic transitions depending on their energy (Rull 2012). Figure 2 sketches some interactions that may happen when an incident energetic photon strikes a molecule. In the mid to far infrared domain, the photon energy of incident light is low and may correspond to the difference between two allowed vibrational levels in the molecular unit. The photon is then absorbed and generates a phonon with the same energy (McMillan and Hofmeister 1988). This is the process analyzed by infrared spectroscopy, which is based on light absorption. Visible light cannot be absorbed through phonon excitation, because the photon energy is much greater than the energy difference between the vibrational states of the molecules. Visible light rather is scattered and excites the molecules to virtual energy states with extremely short lifetimes and quasi-immediate relaxation. Most light scattering is essentially elastic, i.e., the molecule ends up in the same vibrational state as before excitation, and the energy of the scattered light is unchanged. This is Rayleigh scattering (Figs. 1, 2). But a small fraction of the scattering occurs inelastically. In such cases, after scattering, the molecule resides at a higher (energy loss, Stokes scattering) or lower (energy gain, Anti-Stokes scattering) vibrational state than before excitation. Consequently, the photon energy of the scattered light is either decreased or increased with respect to the exciting photon: this is the Raman effect. Note that this photon energy difference corresponds to the specific energy difference between vibrational states of the molecule, thereby imparting molecular information to the Raman signal (Neuville et al. 2014; Mernagh et al. 2018). 


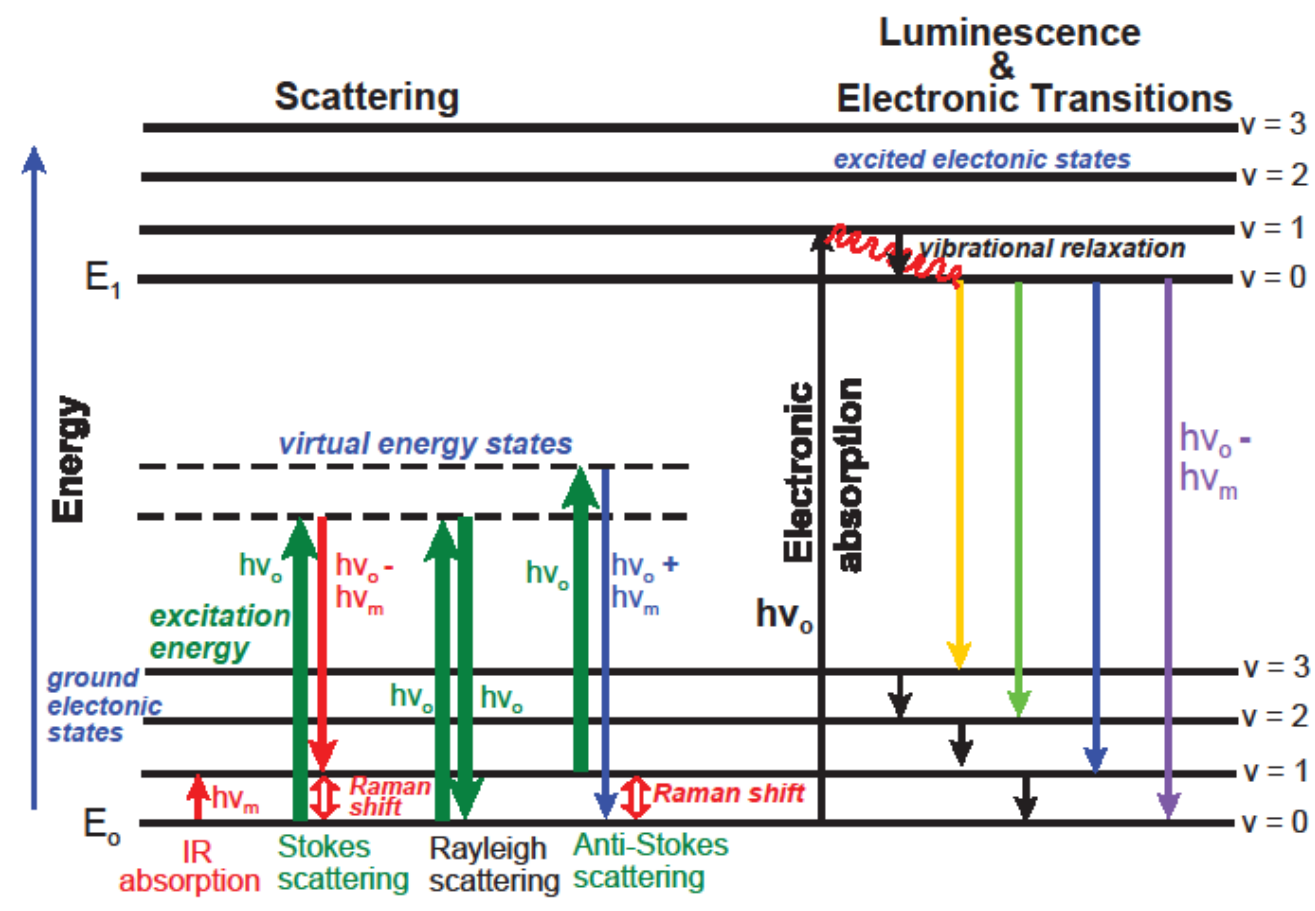

Figure 2: Different types of scattering processes and electronic transitions (x-axis) that may be recorded by the photon detector and the relative energies (y-axis) at which they occur. Stokes, antiStokes, and Rayleigh scattering (also illustrated in Fig. 1) can be seen.

The spectrum acquired encapsulates a combined chemical-structural characterization of the sample. Individual elements are not measured, but the presence and amounts of specific atoms in a phase will affect the vibrational frequency (heavier atoms causing lower frequencies) of a bond and therefore the specific Raman peak position recorded in the spectrum (Fig. 3). In addition to internal vibrations associated with individual molecular units within a crystal (e.g., $\mathrm{CO}_{3}{ }^{2-}, \mathrm{SO}_{4}{ }^{2-}$ , $\left.\mathrm{OH}^{-}\right)$, external vibrational modes, also called lattice vibrations, may be recorded, even in ionically bonded crystals such as fluorite, $\mathrm{CaF}_{2}$. Lattice vibrations/modes arise because the molecules in a crystal - with their specific spacings, symmetry, and bonding - do not vibrate independently of each other. When the displacements of all the atoms, vibrating about their equilibrium positions, are synchronized, they also 
create fundamental lattice vibrations (phonons), which can be monitored in Raman spectroscopy. Such lattice vibrations are also unique features of a specific mineral or other crystalline compound (Fig. 4). In summary, Raman spectra are fingerprints of solids and, like powder XRD patterns, permit identification and characterization of the solid material (Fig. 5); they also can be used to identify the molecular species present in a fluid (Frezzotti et al. 2012; Bodnar and Frezzotti this issue).

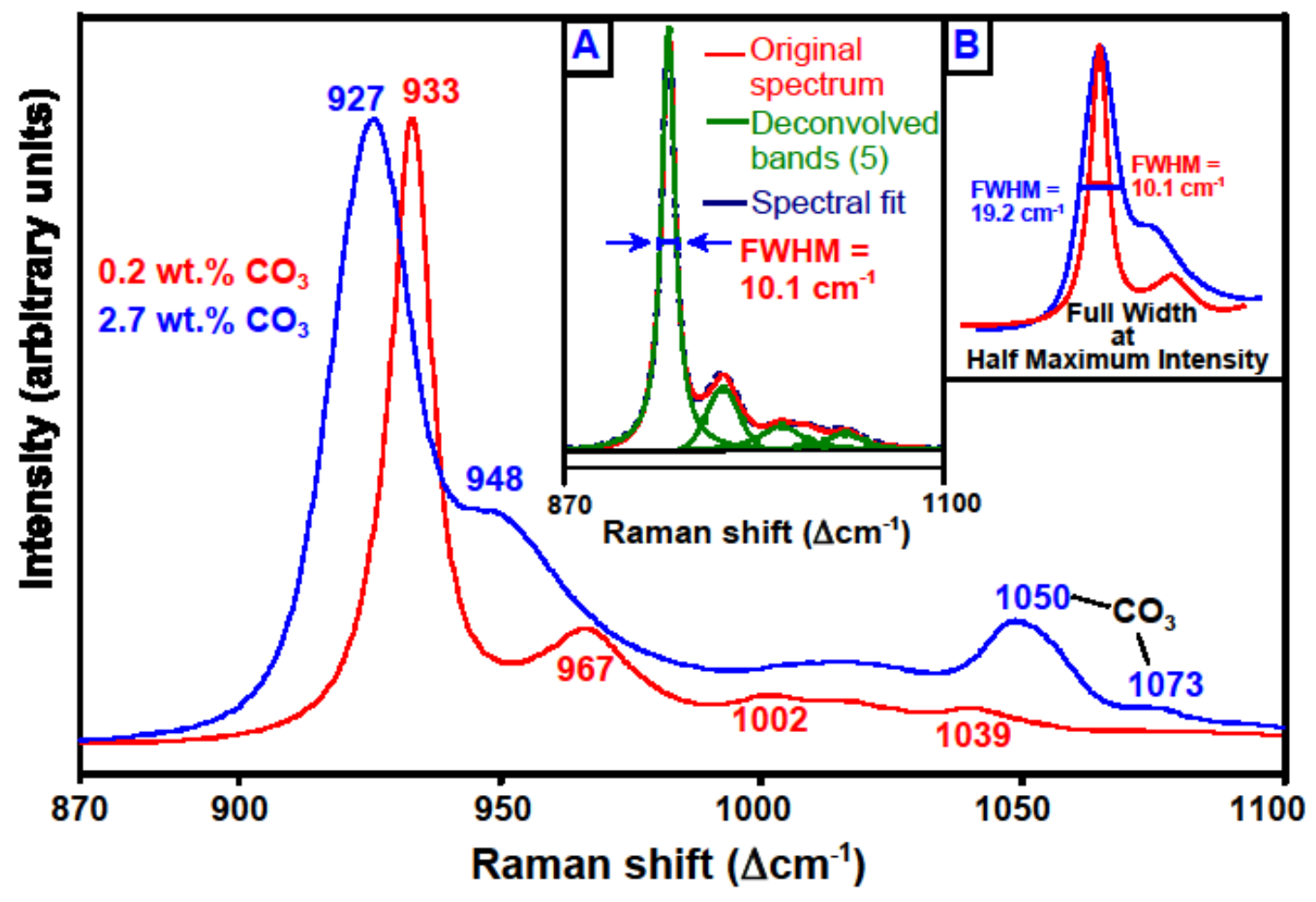

Pasteris_Beyssac_Fig3

Figure 3: Raman spectra of two different synthetic samples of carbonated lead apatite (pyromorphite), differentiated by their amount of $\left(\mathrm{CO}_{3}\right)^{2-}$ substitution, 0.2 or $2.7 \mathrm{wt} \%$. Isomorphous substitution (i.e., solid solution) introduces atomic disorder (thus, band broadening; see inset B) and shifts in peak positions. The complex peaks can be deconvolved into separate, recognized spectral band components (5 such bands shown in inset $A)$. The molecular substituent $\left(\mathrm{CO}_{3}\right)^{2-}$ can be spectroscopically identified and, based on instrument-specific calibration, quantitatively evaluated for concentration. Samples provided by Claude Yoder. 


\section{CAN'S AND CAN'TS}

An enumeration of the Do's and Don'ts in Raman spectroscopy is beyond the scope of this introduction, but a brief explanation of some Can's and Can'ts of Raman are in order, e.g., what kinds of information are obtainable, which samples are most amenable to Raman analysis. Sample preparation is minimal. Polishing is not necessary. No coating on the sample or vacuum chambers are needed. Straightforward Raman identification can be accomplished within seconds. Both crystals and powders of them can be analyzed, but commonly yield differences in their respective signal and background intensities. The caveats typical for techniques using visible light to interact with a sample, however, do pertain. Importantly, the surface state and overall transparency/optical absorption of the sample at the excitation wavelength are key parameters controlling the excitation volume during the analysis.

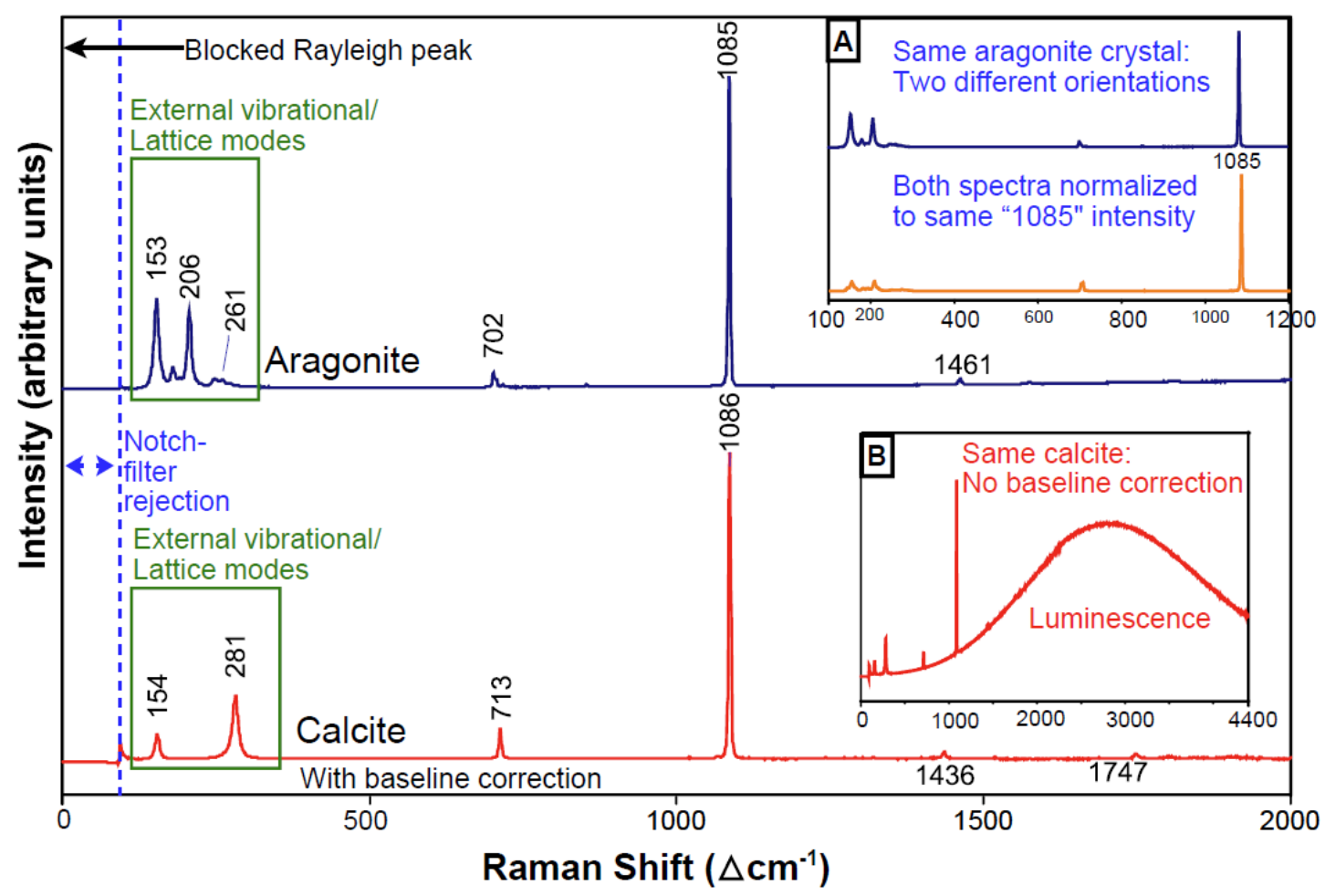


Figure 4: Illustration of Raman spectral differentiation of polymorphs, i.e., $\mathrm{CaCO}_{3}$ phases aragonite and calcite. Note the very strong similarity of the dominant $v_{1}$ peak positions, but visible differences between the other internal vibrational modes and the very obvious difference in the external vibrational/lattice (phonon) modes of the two carbonate phases. Insets $A$ and $B$ illustrate two other confounding factors for one and the same $\mathrm{CaCO}_{3}$ grain. The relative intensity ratios among bands within the spectrum of a given, anisotropic grain will change as the grain is rotated with respect to the laser's plane of polarization (inset A). Many natural minerals luminesce under the laser (inset B), decreasing the apparent signal strength of the mineral. Background correction (seen in bottom, main spectrum) is convenient, but masks the effects of luminescence on the recorded band intensities.

For micro-Raman analysis via a confocal optical system, the beam of the exciting laser is commonly focused to a spot diameter in the range of hundreds of nanometers to a few micrometers, making the spatial scales of information complementary to those of the electron probe microanalyzer. The technique usually is non-destructive, but opaque and highly colored samples may heat up destructively and hydrated minerals may dehydrate due to absorption of the incident laser light (Nasdala et al. 2004; Fries and Steele 2018). Raman is applicable to solids (amorphous to crystalline, mineral or organic), liquids, and gases (Fig. 5). In transparent samples, analyses can be made on targets below the sample surface, e.g., fluid, melt, and mineral inclusions (Bodnar and Frezzotti this issue) or samples in a diamond-anvil cell (Mao and Hemley 1994; Dietrich et al. 2018). Analyses can be made of aqueous components and phases in water, for example, dissolved sulfate and minerals undergoing a reaction. In nanocomposites of organic and inorganic compounds, such as bone, the interwoven phases typically have Raman bands in different parts of the spectrum and thus can be identified individually and without destructive treatment of the sample (Pasteris et al. 2008).

As indicated above, Raman spectroscopy does not identify individual atoms, although identification of the compound itself can reveal much about its chemistry. If the 
Raman spectral identification is diopside, then the sample clearly is dominated by $\mathrm{Ca}$, $\mathrm{Mg}$, $\mathrm{Si}$, and $\mathrm{O}$. Because it is vibrational frequencies of bonds that are being monitored, anything that affects those bonds will affect their frequency and therefore specific peak positions, e.g., isomorphous ion substitution, site vacancies, other defects (Apopei et al. 2017). Indeed, the Raman spectrum is much more sensitive to changes in or differences between compounds than it is to the identity of the element(s) or the specific molecular environment causing the change (Fig. 3).

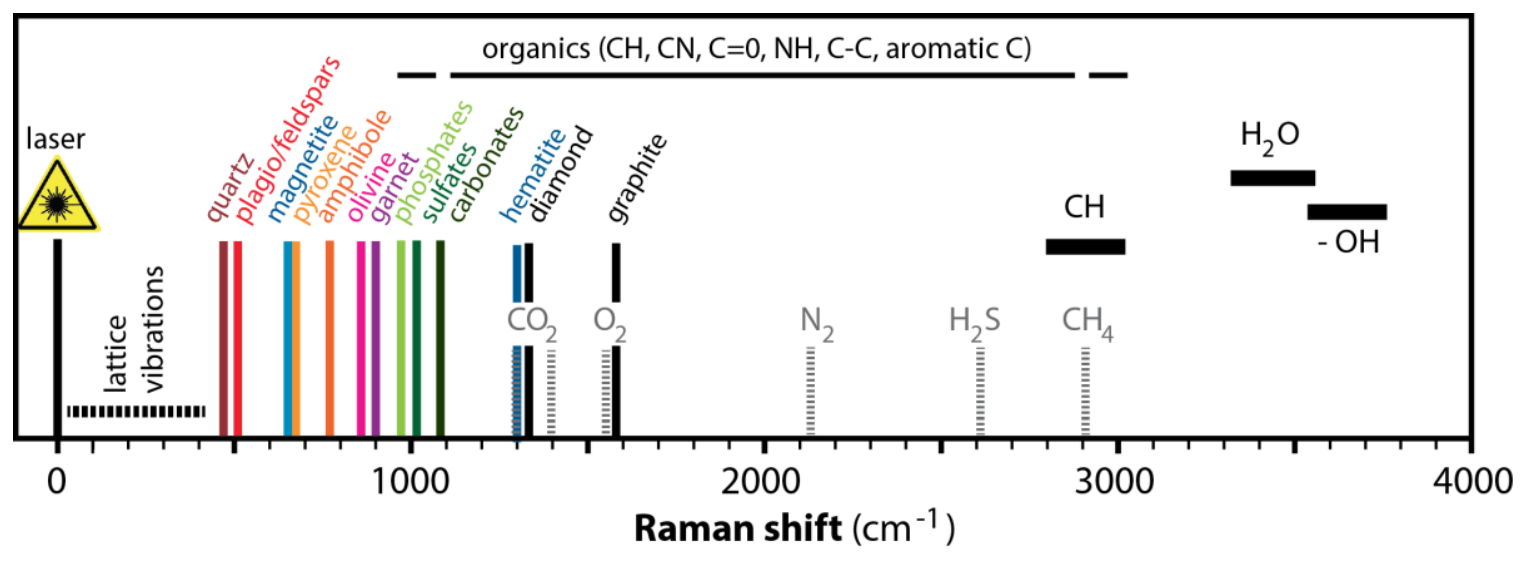

Figure 5: Simplified sketch showing the peak position for one principal peak of various common rock-forming minerals. Peak positions for major gases (vertical gray bars) and organics are also depicted. This is just a sketch to show how the Raman spectrum contains fingerprint information; proper characterization of solids requires the identification of several peaks in most cases.

Various chemical aspects of solid phases, gases, and aqueous species can be explored and characterized by Raman spectroscopy. Because specific bonded entities interact with the incident photons, the Raman spectrum shows the speciation of the atoms involved, e.g., revealing not just that sulfur is dissolved in the water, but that the dominant aqueous sulfur species is $\left(\mathrm{HSO}_{4}\right)^{-}$with lesser concentration of $\left(\mathrm{SO}_{4}\right)^{2-}$ (Benison 2013; Dietrich et al. 2018). The effect on the vibrational frequency of substituting ions of different mass or isotopes of the same element, but different mass, can be calibrated against independently analyzed reference materials. Mixtures of more than two substituents of different masses are more difficult to interpret. The relative state of hydration, hydroxylation, carbonation, etc. (involving covalently bonded species) of a solid phase also can be determined by Raman (Fig. 3). Although polymorphs have the same chemical composition, their contrasts in structure are 
revealed by differences in the number of and/or positions of Raman bands (Figs. 4, 7).

As for all analytical techniques, Raman spectroscopy has its drawbacks and limitations. As suggested above, not all compounds are Raman active (i.e., produce spectral peaks), and some have much stronger scattering efficiencies than others, e.g., diamond (very strong) vs. nanocrystalline iron oxides (weak). In particular, many natural samples have impurities (e.g., defects, trace elements, organics) that can generate a luminescence signal when excited (Figs. 2, 4). This luminescence signal may have various expressions (e.g., broad bands to continuous background, as exhibited by $\mathrm{Mn}^{2+}$ substitution in calcite, Fig. 4; discrete lines, as exhibited by rareearth elements in Raman-active or -inactive minerals) and may be so intense that the Raman signal becomes subsumed by and often indistinguishable from the luminescence band and/or background (Fig. 4 inset B). Narrow, laser-induced emission lines can be mistaken for Raman bands, thereby causing confusion and possible errors in sample identification. There are also concerns of sample alteration (e.g., melting, oxidation, chemical breakdown) by laser heating (Nasdala et al. 2004) and heat-induced downshifting of Raman bands (Bryant et al. 2018).

Aspects of the specific instrument being used, as well as the physics of the Raman effect, put constraints on the type of information retrievable and the specificity of the interpretation of the spectra. The spectrum shows only what the focused laser was able to "sample", especially for highly confocal instruments. In opaque or strongly light-scattering samples (e.g., fine-grained powders), the spectrum will be dominated by the surficial species/phases. The latter two factors make it difficult to readily determine with a Raman microprobe the quantitative ratio of solid phases in a mixture, because it is a scattering signal (Raman) rather than a volume-integrating absorption signal (transmission IR) that is evaluated. In individual, non-isometric crystals, the orientation of the crystal with respect to the plane of polarization of the incoming laser will affect the relative intensities of (some of) the Raman peaks (Fig. 4 inset A), as will inherent strain in the material. 


\section{INTERPRETABLE ASPECTS OF A RAMAN SPECT RUM}

The standard presentation of a Raman spectrum shows scattering intensity on the vertical axis and wavenumber shift on the horizontal axis (Fig. 1). More specifically: The intensity data initially are recorded as the number of Raman-scattered photons detected per unit time, the exact values of which depend on multiple sampling and instrumental factors. The vertical axis therefore is typically labeled with "arbitrary units" and indicates the relative peak intensities across a single given spectrum (Fig. 3). The horizontal axis is based on wavenumber, calculated as $1 / \lambda$ (where $\lambda$ units are in $\mathrm{cm}$ ), i.e., "wavenumber, $\mathrm{cm}^{-1}$." It is actually the difference in wavenumber values between that of the incoming photons (from the laser) and the scattered photons that is plotted; the units displayed on the $\mathrm{x}$-axis are the absolute wavenumber value of the laser light minus the absolute wavenumber value of the scattered photons, i.e., the socalled Raman shift, in $\Delta \mathrm{cm}^{-1}$. There are two major benefits to this mode of presentation: 1) Raman band positions are labeled identically regardless of which laser wavelength is used for excitation, and 2) the $\Delta \mathrm{cm}^{-1}$ wavenumber values (listed simply as $\mathrm{cm}^{-1}$, in typical practice) in a Raman spectrum match the $\mathrm{cm}^{-1}$ wavenumber values in the IR spectrum of the same material for those vibrational modes that are both Raman- and IR-active (Fig. 2).

Several distinct characteristics of a Raman spectrum can be examined for chemicalstructural information: number of peaks, peak position, peak shape, and relative peak area (Fig. 3). The number of peaks seen in a Raman spectrum (for solids, typically a smaller number than would be calculated by group theory) indicates the number of Raman-active vibrational modes (i.e., internal molecular modes, crystal field splittings, librations, and lattice /external vibrational modes). Lattice modes/phonons in some cases can be viewed directly in low-wavenumber Raman spectroscopy (Fig. 4). The symmetry of the bonded component as well as that of the over-all structure control the number of peaks, which is larger for the less symmetric structures. The peak position indicates the vibrational frequency of the specific mode 
within its over-all structure, thereby reflecting the bond's strength and the atomic masses of the bonded atoms. Thus, few-wavenumber offsets between the peak positions obtained on the same instrument for a pure end-member compound and for a geological sample of the same mineral/compound indicate some amount of solid solution present in the geological sample (Fig. 3).

The shape of the "peak" (the as-observed signal, i.e., the recorded "hump" in the raw spectrum) is also revealing. Particularly if the observed peak is asymmetric, it likely comprises two or more underlying Raman bands, but the asymmetry also may reflect very small particle size or a misalignment between the spectrometer and detector. Such spectra can be better characterized with instruments of higher spectral resolution. With appropriate software, one can deconvolve the peak into separate, spectroscopically recognized bands that are characteristic of specific vibrational modes (Fig. 3, inset A). Even if the peak appears symmetric, its FWHM value (quantified as full band width assessed at half intensity/height maximum) should be examined, as should the FWHM of constituent bands. For several samples of the same compound/mineral, but which formed under different conditions (e.g., temperature, cooling rate, compositional complexity, grain size), the peak widths typically will differ. The narrower the peak, the more atomically well ordered the solid is (Fig 3, inset B). For example, rapid cooling from a high-temperature melt and spontaneous precipitation from a strongly supersaturated solution at low temperature can lead to broader bands than for crystals that formed slowly and in equilibrium with their host melt or solution (Maksimuk et al. 2005). There are cases in which relative peak heights or areas can be used (especially in conjunction with a set of independently analyzed reference standards) to determine proportions of components within the same phase. In most cases, peak areas (or areas of deconvolved bands) are recommended as more reproducible than heights for quantification, e.g., the amount of carbonate substitution in apatite [ratio of spectral bands for $\mathrm{CO}_{3} /\left(\mathrm{CO}_{3}+\mathrm{PO}_{4}\right)$ ] (Awonusi et al. 2007), the degree of crystallinity of graphitic carbon [relative contribution of defect bands] (Wopenka and Pasteris 1993; Beyssac et al. 2002), and the molar fractions of $\mathrm{CH}_{4}, \mathrm{~N}_{2}$, and $\mathrm{CO}_{2}$ in the gas phase of a fluid inclusion (Frezzotti 
et al. 2012). Importantly, quantitative use of spectral data (e.g. FWHM, peak area ratios) from different instruments should be done only after correction for the instrumental response function (Nasdala and Schmidt this issue).

Over the past few decades, several different groups have developed and made available on-line their Raman spectral databases, representing spectra of wellcharacterized minerals. The best known of these is the RRUFF Raman, IR, and XRD database (http://rruff.info/about/about general.php), which contains over 14,000 high-resolution Raman spectra and their accompanying downloadable data files. The majority of the minerals have been confirmed by XRD and/or chemical analysis. The database is searchable by mineral name and by major elements (Lafuente et al. 2015).

\section{RAMAN HYPERSPECTRAL MAPPING}

Over the last decade, a very important methodological development has been Raman hyperspectral mapping, which opened Raman to the imaging of internal microtextures of minerals and mineral assemblages (Nasdala et al. 2012; Fries and Steele 2018). Raman mapping consists in plotting in two, or even three, dimensions a spectral parameter extracted from a collection of spectra precisely located on the sample. The experimental principle is very simple: move the sample with constant step in $\mathrm{X}$ and $\mathrm{Y}$ directions and record a spectrum at each position. Three-dimensional mapping may be done when using transparent samples but is rather challenging to properly process and interpret. In general, data processing will consist in extracting the appropriate information from each spectrum and plotting it in 2D or 3D maps. Depending on the purpose of the map, this information could be 1) a peak position for strain patterns or compositional variations in minerals, 2) the presence or absence of a representative peak for each mineral/gas for mapping mineral/gas assemblages in a rock or a fluid inclusion, or 3) the FWHM of a selected peak for mapping defects in minerals, among many other possibilities. Please see examples in other articles of this issue. Raman mapping can be very useful to detect and locate a specific feature (e.g., mineral phase, fluid inclusion, organics) in a sample (including 
powders) and/or to retrieve a very large spectral dataset acquired on a specific sample in order to derive multiple types of statistics.

For a long time, Raman hyperspectral mapping suffered from the very long duration of the spectral acquisition, but recent developments in hardware and, overall, software make possible the generation of maps encompassing thousands of spectra within minutes. Manufacturers of Raman systems have deployed various strategies, such as line-scan mapping, dynamic mapping involving synchronization of sample displacement with data reading on the detector, and dynamic and continuous autofocus, which makes Raman mapping an easy-to-use technique with modern instruments. Importantly, the spatial resolution of Raman mapping is mostly controlled by the sampling step distance and the lateral resolution of the laser spot for each analysis, both of which typically are on the order of a micrometer. Of course, all methodological traps inherent to Raman spectroscopy remain, and there are more specific drawbacks to mapping depending on the instrument used and sample analyzed (Nasdala et al. 2012). The relative maturity of the technique now permits most of these problems to be overcome, if the operator is aware and cautious. All the parameter choices made to generate a Raman image have to be clearly specified. This includes not only the data processing (e.g., details on peak-fitting procedure, methodology used for principal-component analysis), but also the link between the scientific information mapped (e.g., mineralogy, strain distribution, mineral or gas composition) and the raw spectral data (e.g., peak identification, FWHM, peak position, or area ratio). Two examples of necessary considerations of such links are the desire to know stress, when Raman spectra reveal strain (Korsakov et al. this issue), and the desire to determine (fluid) pressure, when Raman spectra sensitively monitor density (Bodnar and Frezzotti this issue). 


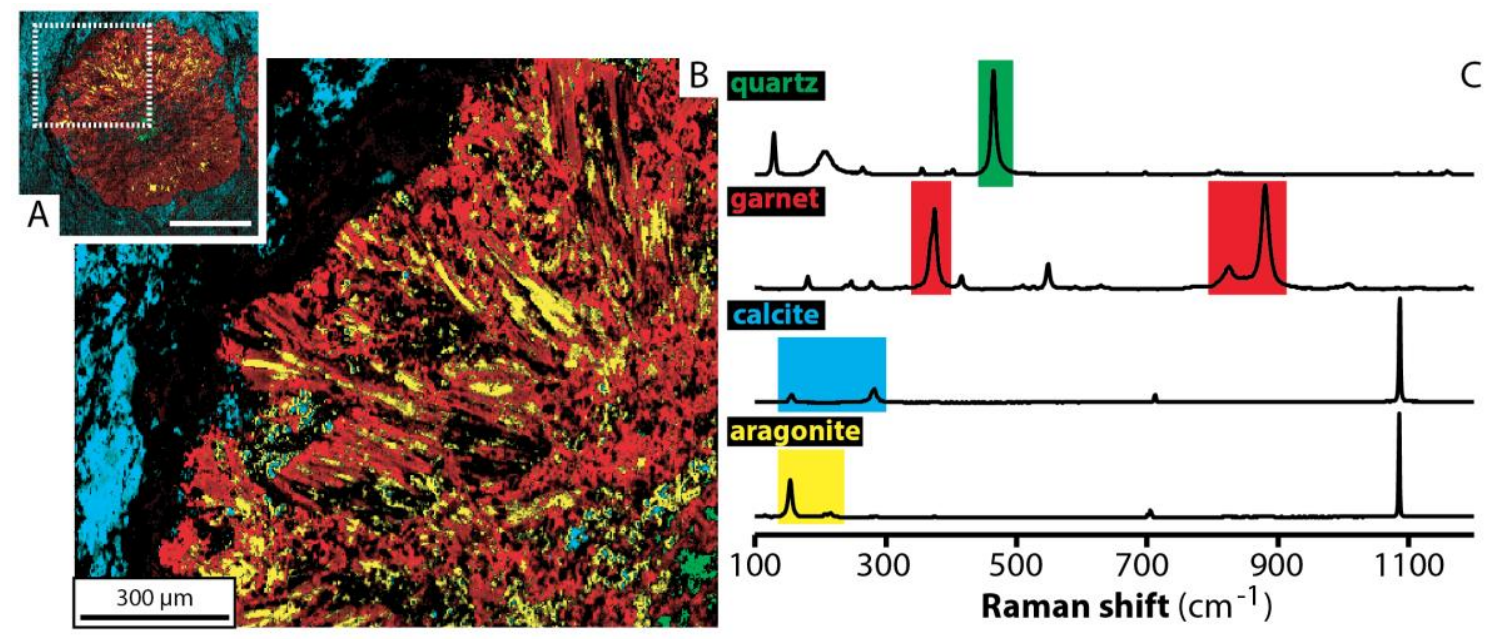

Figure 6: Raman map of a garnet crystal containing various mineral inclusions: quartz and calcite in the core, fibrous aragonite in the rim. The rock matrix is composed of calcite and graphitic carbon (here in black). The sample comes from Alpine Corsica (France). A-general Raman map of a garnet crystal (scale bar $1 \mathrm{~mm}, 10 \times 11 \mu \mathrm{m}$ sampling step). B-zoom of the box in A (3.3 $3.3 \mu \mathrm{m}$ sampling step). $C$-Color codes used on the map. Reference spectra used for the map: colored vertical bars indicate the specific peak(s) used to identify each mineral phase. Note that external/lattice vibrations at low Raman shifts were used to distinguish calcite from aragonite. Modified after Chopin et al. (2008).

Figure 7 illustrates the Raman mapping of aragonite-garnet intergrowths in an impure marble. A similar map of such a mineral assemblage at similar resolution easily could be obtained by techniques based on X-ray spectroscopy, such as SEMEDX (scanning electron microscopy-energy dispersive X-ray spectroscopy) or EPMA (electron probe microanalysis), but the ability of Raman to distinguish mineral polymorphs is invaluable here. Techniques based on X-ray spectroscopy actually would map Ca-carbonate as a high-Ca phase, but would not distinguish the polymorphs calcite and aragonite. Such distinction is possible by Raman, as by detecting lattice vibrations specific to each mineral structure (Fig. 4). Raman mapping has become so popular that it is now used in virtually all field of Earth sciences as attested by the examples provided throughout the following articles.

\section{IN THIS ISSUE}

In the articles in this issue of Elements, our goal is to highlight several of the Earth and Planetary Science fields in which Raman analysis, dominantly via Raman microprobe systems, has had wide-ranging impacts. The selection of topics was difficult, but our 
intention is to demonstrate a sufficient number and diversity of applications (with their inherent "successes," challenges, and pitfalls) to enlighten readers of Ramanbased studies and to encourage more geoscientists to apply this technique in both well-accepted and new ways. Bodnar and Frezzotti review the application of Raman for studying fluid and melt inclusions. This is truly an historical but still continuously developing field of application, as Raman spectroscopy is one of the very few techniques allowing in situ characterization of such tiny objects. Nasdala and Schmidt show the versatility and rich information offered by Raman spectroscopy to mineralogy and geochemistry. Korsakov et al. discuss the variety of geological information that can be extracted from rocks, thanks to Raman spectroscopy, to better understand their tectonic and metamorphic history. Steele et al. show how Raman spectroscopy has played and still plays a key role in the emerging interdisciplinary fields of biomineralogy and biogeochemistry. Lastly, Beyssac discusses some new trends in Raman spectroscopy for geoscientists from applications in the laboratory to the exploration of Mars.

\section{ACKNOWLEDGMENTS}

The authors wish to thank Lutz Nasdala and an anonymous reviewer for their detailed, insightful comments and Nancy Ross for her helpful editorial handling. Raman instruments at IMPMC were funded by the city of Paris, Sorbonne Université, and CNRS. JDP thanks John Freeman for numerous spectroscopy discussions over the years and the U.S. Department of Energy and Washington University in St. Louis for the Raman instrument used.

\section{REFERENCES CITED}

Adar F (2001) Evolution and revolution of Raman instrumentation--Application of available technologies to spectroscopy and microscopy. In: Lewis IR, Edwards HGM 
(eds) Handbook of Raman Spectroscopy, Marcel Dekker, Inc., New York, pp 11-40

Apopei AI and 5 coauthors (2017) The determination of the Sb/As content in natural tetrahedrite-tennantite and bournonite-seligmannite solid solution series by Raman spectroscopy. Mineralogical Magazine 81: 1439-1456

Awonusi A, Morris MD, Tecklenburg MMJ (2007) Carbonate assignment and calibration in the Raman spectrum of apatite. Calcified Tissue International 81: 4652

Benison KC (2013) Acid saline fluid inclusions: Examples from modern and Permian extreme lake systems. Geofluids 13: 579-593

Beyssac O, Goffe B, Chopin C, Rouzaud JN (2002) Raman spectra of carbonaceous material in metasediments: A new geothermometer. Journal of Metamorphic Geology 20: 859-871

Bryant RN, Pasteris JD, Fike DA (2018) Variability in the Raman spectrum of unpolished growth and fracture surfaces of pyrite due to laser heating and crystal orientation. Applied Spectroscopy 72: 37-47

Cardell C, Geurra I (2016) An overview of emerging hyphenated SEM-EDX and Raman spectroscopy systems: Applications in life, environmental and materials sciences. Trends in Analytical Chemistry 77: 156-166

Chopin C, Beyssac 0, Bernard S, Malavieille I (2008) Aragonite-garnet intergrowths in eclogite-facies marble, Corsica. European Journal of Mineralogy 20: 857-865

Chou I-M, Wang A (2017) Application of laser Raman micro-analyses to Earth and planetary materials. Journal of Asian Earth Sciences 145: 309-333

Dietrich M, Behrens H, Wilke M (2018) A new optical cell for in situ Raman spectroscopy, and its application to study sulfur-bearing fluids at elevated pressures and temperatures. American Mineralogist 103: 418-429

Dubessy J, Caumon M-C, Rull F, Sharma S (2012) Instrumentation in Raman spectroscopy: Elementary theory and practice. In: Dubessy J, Caumon M-C, Rull F (eds) Raman Spectroscopy Applied to Earth Sciences and Cultural Heritage, European Mineralogical Union and Mineralogical Society of Great Britain \& Ireland, London, pp 83-172

Frezzotti ML, Tecce F, Casagli A (2012) Raman spectroscopy for fluid inclusion analysis. Journal of Geochemical Exploration 112: 1-20 
Fries MD, Steele A (2018) Raman spectroscopy and confocal Raman imaging in mineralogy and petrography. Springer Series in Surface Sciences 66: 209-236

Lafuente B, Downs RT, Yang H, Stone N (2015) The power of databases: The RRUFF project. In: Armbruster T, Danisi RM (eds) Highlights in Mineralogical Crystallography, W. De Gruyter, Berlin, pp 1-30

Maksimuk M and 4 coauthors (2005) Influence of the cooling rate on lowtemperature Raman and infrared-reflection spectra of partially deuterated K(BEDT-TTF $)_{2} \mathrm{Cu}\left(\mathrm{N}(\mathrm{CN})_{2}\right) \mathrm{Br}$. Synthetic Metals 149: 13-18

Mao H-K, Hemley RJ (1994) Ultra-high-pressure transitions in solid hydrogen. Reviews of Modern Physics 66: 671-692

McMillan PF, Hofmeister AM (1988) Infrared and Raman spectroscopy. In: Hawthorne FC (ed) Spectroscopic Methods in Mineralogy and Geology, Mineralogical Society of America, Chantilly, Virginia, pp 99-159

Mernagh TP and 4 coauthors (2018) Using infrared and Raman spectroscopy to analyze gas-solid reactions. In: King PL, Fegley B, Seward T (eds) High Temperature Gas-Solid Reactions, Mineralogical Society of America, Chantilly, VA, pp 177-228

Nasdala L, Beyssac O, Schopf JW, Bleisteiner B (2012) Application of Raman-based images in the Earth sciences. In: Zoubir A (ed) Raman Imaging, Springer Nature, Cham, Switzerland, pp 145-187

Nasdala L, Smith DC, Kaindl R, Ziemann MA (2004) Raman spectroscopy: Analytical perspectives in mineralogical research. EMU Notes in Mineralogy 6: 281-343

Neuville DR, de Ligny D, Henderson GS (2014) Advances in Raman spectroscopy applied to Earth and materials sciences. Reviews in Mineralogy and Geochemistry 78: 509-541

Pasteris JD, Wopenka B, Valsami-Jones E (2008) Bone and tooth mineralization: Why apatite? Elements 4: 97-104

Rull F (2012) The Raman effect and the vibrational dynamics of molecules and crystalline solids. EMU Notes in Mineralogy 12: 1-60

Wopenka B, Pasteris JD (1993) Structural characterization of kerogens to granulitefacies graphite: Applicability of Raman microprobe spectroscopy. American Mineralogist 78: 533-557 